\title{
Make new friends, but keep the old: towards a transdisciplinary and balanced strategy to evaluate Good Environmental Status
}

Submitted 20 March 2015; accepted 7 April 2015; first published online 6 May 2015

The Marine Strategy Framework Directive (MSFD), adopted by the European Union in 2008, has as its main goal: 'Achieve Good Environmental Status (GES) by 2020'. The MSFD defines 11 descriptors of GES (http://ec.europa.eu/ environment/marine/good-environmental-status/index_en.htm). This list is a strong contribution to the (refreshing and innovative) view that in order to assess the quality of the environment, biodiversity and ecosystem functioning must be incorporated as pillars of GES.

We have to move beyond simplistic descriptors (e.g. the concentration of contaminants, or the number of E. coli) and thresholds that were previously used to evaluate GES. These were failing to capture the complexity of environments and ecosystems. For example, species are exposed to a range of environmental conditions and understanding the interactions between different environmental drivers is a challenge. Even if each individual driver is below a given threshold, drivers in combination might affect the quality of the environment in a significant way.

The revolution of the MSFD and the GES descriptors is very simple: rather than simply looking at simplistic thresholds, we should consider consequences for organisms and ecosystems. GES focused on two main pillars: 'biodiversity' and 'ecosystem functioning'. As a consequence, biology in its whole complexity should be included to ensure that 'biodiversity is maintained' (descriptor 1) or maintenance of 'sea floor integrity that ensures functioning of the ecosystem' (descriptor 6).

The scientific community has established a sophisticated observation system that employs state of the art instrumentation to obtain information about the values of important descriptors (e.g. currents, temperature, $\mathrm{pH}$, chlorophyll etc.). We have satellites, buoys, gliders, submersibles as well as sophisticated ship-board technology that together extract and organize enormous amounts of data. However, these data are not sufficient to evaluate biological functionality. To fit the requirements of the GES descriptors, we need direct biological measurements and a strong mechanistic understanding of the relationship between the physicochemical environment and biological responses across levels of organization. This can only be achieved through substantial strategic investments in field and experimental biological sciences, including neglected fields such as taxonomy. This will redress the imbalance and indeed bring considerable added value to the extensive physico-chemical datasets available. For example, it is worrying that expertise in taxonomy is vanishing in the European Union, and even simple identification can be difficult. While automated identification with genetic bar coding is an interesting option, the barcoding of life still requires the expertise of classic taxonomists to ensure that a barcode is applied to a specimen that is correctly identified. Once we have established exactly what we have living in the ecosystem we can then begin to consider the functionality of those organisms. It is this that will ultimately determine the sustainability, resilience and sensitivity of the ecosystem.

In the same way, field and experimental biologists should benefit from closer contacts with monitoring programmes and modellers. Monitoring programmes can provide valuable information on species' abiotic niches; modellers can provide scenarios for future changes and identify key parameters that would require experimentalists' attention. In return, biologists can help the design of new monitoring programmes to define biologically relevant spatio-temporal scales as well as requested levels of accuracy.

A new era of collaboration is open and the rich heritage and traditional inter-disciplinarity of our Coastal Marine Stations offers an exciting network of infrastructural platforms ready and able to facilitate this (http://www.marsnetwork.org/). We are calling for increased communication between the different fields involved. Technologists must design new sensors and monitoring approaches that are in line with the requirements of GES, biologists and ecologists must revive those disciplines that have been neglected or abandoned. This can only be achieved through a more balanced distribution of resources and a clear understanding of the culture and history of the different disciplines.

Assessment of the full complexity of GES in the context of an ever-changing world is a challenging task and associated with large uncertainties and many knowledge gaps. However, uncertainties should never be an excuse for inaction. In the face of global environmental change, we can now start 
the process of bridging old and new disciplines, monitoring field and experimental biology, modelling and many more to develop the tools essential for society's needs. For example, we can develop ecosystem 'risk maps' based on our best present knowledge that identify those regions most at risk by combining chemistry (e.g. rate of change), geology (e.g. habitat structure), taxonomy (e.g. biodiversity, species uniqueness), physiology (e.g. species sensitivity), ecology (e.g. ecosystem structure), evolution (e.g. adaptation potential), socio-economics (e.g. dependency), etc. This will lead to an iterative process, each discipline informing all others and defining priorities. There can be no doubt either that this approach offers significant potential as the community moves forward, as it must, with the development of networks of Marine Protected Areas (e.g. http://www.coconet-fp7.eu/). Initiatives such as the newly established transdisciplinary and 'bottom-up' consortium Euromarine (http://www.euromarinenetwork.eu/) is a significant step in the right direction.

Very interesting times lie ahead for us all.

\section{Correspondence should be addressed to:}

M. Thorndyke

Royal Swedish Academy of Sciences and Göteborg University, Sweden

email: mike.thorndyke@bioenv.gu.se

Ferdinando Boero $^{1}$, Sam Dupont ${ }^{2}$

and Michael Thorndyke ${ }^{3}$

${ }^{1}$ University of Salento, CNR-ISMAR, Italy, ${ }^{2}$ University of Gothenburg, Sweden, ${ }^{3}$ Royal Swedish Academy of Sciences and Göteborg University, Sweden 